\title{
A generic approach for the catalytic reduction of nitriles
}

\author{
Stephen Caddick, ${ }^{\text {a,* }}$ Duncan B. Judd, ${ }^{\mathrm{b}}$ Alexandra K. de K. Lewis, ${ }^{\text {a }}$ Melanie T. Reich ${ }^{\mathrm{a}}$ \\ and Meredith R. V. Williams ${ }^{\mathrm{a}}$ \\ ${ }^{a}$ The Chemistry Laboratory, University of Sussex, Falmer, Brighton, East Sussex BN1 9QJ, UK \\ ${ }^{\mathrm{b}}$ GlaxoSmithKline, New Frontiers Science Park (SC1), Third Avenue, Harlow, Essex CM19 5AW, UK
}

Received 17 March 2003; revised 9 May 2003; accepted 29 May 2003

\begin{abstract}
The scope of nickel boride mediated reduction of nitriles has been extended further to allow the preparation of Boc protected amines via a mild catalytic process. It is noteworthy that the toxicity of this procedure is greatly reduced due to its catalytic nature in nickel(II) chloride used in combination with excess sodium borohydride. The protocol is marked by its resilience towards air and moisture and hence an easy and general practical protocol. (C) 2003 Elsevier Science Ltd. All rights reserved.
\end{abstract}

\section{Introduction}

With increasing demand for generic procedures for solution phase chemistry and a broad range of commercially available nitriles, it became desirable to devise an improved protocol for the reduction of the surprisingly unreactive cyano group. Although the reduction of nitriles to yield amines has found endless application in synthetic organic chemistry over the years, ${ }^{1}$ most procedures involve the use of strong reducing agents and hence lack selectivity and general procedures. The most commonly used conditions are strong hydride donors, such as lithium aluminum hydride, or catalytic hydrogenation. ${ }^{1}$ Diborane mediated reduction has also found numerous applications and works particularly well in the presence of nickel(II) chloride. $^{2}$ Sodium borohydride, a milder, easier-to-handle reagent, would offer better scope for functional group compatibility, but is generally not strong enough to bring about reduction of the cyano group, ${ }^{1}$ although exceptions do exist. ${ }^{3}$ Numerous examples can be found in the literature, demonstrating the enormous change in behaviour of this reagent upon the addition of e.g. transition metal salts, which allows fine-tuning of the reactivity of the metal hydride. ${ }^{4,5}$ The best studied and most reliable of such additives are nickel and cobalt salts. ${ }^{2,6,7}$ Schlesinger et al. ${ }^{8}$ first reported the preparation of cobalt(II) boride and nickel(II) boride under aqueous conditions from sodium borohydride in 1949. Traditionally these metal borides, especially nickel(II) boride, ${ }^{9}$ are used as catalysts for hydrogenation, ${ }^{10,11}$ but have more recently found numerous applications as reducing agents in their own right. ${ }^{12-15}$ Most metal assisted borohydride reductions employ stoi-

Keywords: catalytic; nickel boride; reduction; nitriles.

* Corresponding author. Tel./fax: +44-1273678734;

e-mail:s.caddick@sussex.ac.uk chiometric quantities of the transition metal salt and an excess of the metal hydride converting groups such as cyano and nitro. ${ }^{12-15}$ The precise nature of the species formed upon the reaction of the metal salt with the borohydride depends very much on the exact reaction conditions. ${ }^{10,16}$ With uncertainties regarding the structure and stoichiometry of the species formed, ${ }^{17}$ development of general and reliable reduction procedures with predictable functional group compatibility has not been possible so far. In fact, disagreements on this topic can be found throughout the literature, concerning for example the relative strengths of the reducing systems formed from cobalt and nickel species with borohydride. ${ }^{12,18}$ Although some examples do exist where catalytic quantities of nickel boride have been employed to reduce nitro groups and heterocycles, a general protocol has thus far not been developed. ${ }^{12,19,20}$

\section{Results and discussion}

Herein, we report on a generic reduction protocol for nitriles employing catalytic quantities of nickel(II) chloride with excess sodium borohydride to facilitate the formation of Boc protected amines. Previous work in our laboratory had led to the conclusion that stoichiometric amounts of nickel boride, a cheap, simple to prepare, air-stable, amorphous substance, could mediate reduction of the cyano group in the presence of excess sodium borohydride in methanol. ${ }^{21}$ Complete conversion was achieved in clean reactions to give secondary amines. In order to isolate primary amines treatment with acetic anhydride or di-tert-butyl dicarbonate in a one-pot reaction was required to prevent dimerisation, a well known side reaction that can occur during the reduction of nitriles unless the reaction conditions are carefully adjusted. $9,13,19,21,22$ This method of trapping the primary 
amine is analogous to that employed in the hydrogenation of nitriles. ${ }^{23}$ It should be noted that Khurana et al. ${ }^{24}$ have recently reported the reduction of nitriles to primary amines with little or no secondary amine detected by changing to anhydrous conditions and performing the reduction with stoichiometric amounts of nickel(II) chloride, using a limited amount of sodium borohydride in ethanol. In contrast our previously reported procedure requires a reasonably large excess of metal hydride in order for the reaction to proceed to completion, strengthening the suspicion that small changes in conditions result in the formation of a fundamentally different nickel species.

Intrigued by the nature of the reagent generated we investigated the use of nickel boride as a catalyst for sodium borohydride mediated reduction ${ }^{12,19,20}$ rather than as a reducing agent in its own right. Reduction of benzyl cyanide utilising as little as 0.1 equiv. of nickel(II) chloride brought about complete conversion to $N$-Boc phenethylamine $\mathbf{2 b}$ in $96 \%$ isolated yield (cf. 1 equiv. $\left.\mathrm{NiCl}_{2}, 99 \%\right)^{21}$ (Scheme $1 ; \mathrm{R}=\mathrm{Bn}$ ). This result clearly demonstrates that the procedure can be carried out catalytically, however, it should be noted that in the case of $N$-acetyl phenethylamine incomplete conversion was observed $(38 \%)$ for the catalytic process (cf. 1 equiv. $\mathrm{NiCl}_{2}, 94 \%$ ), ${ }^{21}$ which one can speculate is the result of rapid catalyst decomposition in the presence of the acetic acid generated during the reaction. Consequently our investigations focused on the standardisation of this catalytic procedure for the reduction of nitriles to Boc protected amines. The requirements for this transformation are: (i) the starting nitrile needs to be present in the reaction mixture before formation of the metal boride, (ii) fresh nickel boride prepared from nickel(II) chloride hexahydrate and sodium borohydride in methanol, ${ }^{25}$ (iii) a large excess of sodium borohydride and (iv) a trapping agent, essential to circumvent the formation of dimers. The most notable advantages of the catalytic procedure over the stoichiometric are: (i) reduced toxicity, (ii) easy removal of nickel boride with diethylenetriamine during work-up and (iii) the less vigorous nature of the reaction. This catalytic reduction was carried out on an array of 30 different nitriles (Table 1). Although functional group compatibility is limited, compared with previously reported methods it presents an improvement and most importantly is consistent and reliable. Yields are moderate to good, but the cleanliness of the reactions has been exceptional, with no further purification necessary after work-up. Subsequent deprotection of the Boc amines to yield the corresponding amine hydrochloride salts was achieved following a wellknown literature procedure employing anhydrous hydrogen chloride in ethyl acetate (aromatic compounds: 60-80\%, aliphatic compounds: $50-60 \%){ }^{26}$

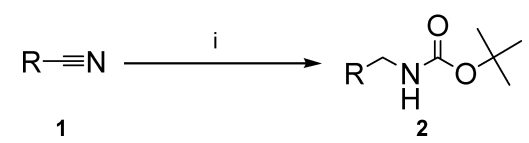

Scheme 1. Reagents and conditions: (i) $\mathrm{NiCl}_{2} \cdot 6 \mathrm{H}_{2} \mathrm{O}$ (0.1 equiv.), $\mathrm{NaBH}_{4}$ (7 equiv.), $\mathrm{Boc}_{2} \mathrm{O}$ (2 equiv.), $\mathrm{MeOH}, 0^{\circ} \mathrm{C}$ to rt, $15 \mathrm{~h}$.

Benzonitriles and benzyl cyanide derivatives (entries 1, 2, 7, and 8) generally undergo this reduction in better yields than aliphatic nitriles (entries 4-6). Selectivity is moderate and although some groups are affected by the reaction conditions in these cases clean reduction of that functionality occurs with no other side-products observed. Nitro groups, as suggested by literature precent, ${ }^{4,19,20,27}$ were not compatible with this procedure and were cleanly converted into the corresponding amine and isolated as the Boc protected derivative (entries 10 and 11). It should be noted, however, that reduction of nitro groups by this type of reducing system utilising cobalt boride has previously been shown to be very sensitive and careful choice of temperature has been reported to allow reduction of a cyano group leaving the nitro group unaffected. ${ }^{13}$ Sodium trifluoroacetoxy borohydride has also been shown to reduce nitriles without affecting nitro groups. ${ }^{28}$ Isolated double bonds were hydrogenated by this metal boride system (entries 14 and 15), as previously reported in the literature. ${ }^{4,29}$ In some cases, however, when cobalt salts were employed no reduction of the double bond occurred. ${ }^{13}$ As documented in the literature ${ }^{4,30}$ a ketone was found to be reduced to the corresponding secondary alcohol (entry 28) in the presence of a boride species and excess borohydride. Pyridine derivatives display at worst moderate yields (entries 12 and 24), consistent with the lower yields reported for these species. ${ }^{13,14}$ Other heterocycles do not appear to be affected by this reaction (entries 16, 17, and 22). ${ }^{12}$ Neither hydroxyl groups nor amines interfere with the reaction (entries 16 and 26), merely Boc protection may occur (entry 9). Other functional groups found to survive the conditions intact were aromatic halides (entries 19,20, and 23), a tert-butyl ester (entry 27) and a sulfonamide (entry 29). Furthermore, primary and secondary amides survive this transformation, albeit in moderate yield (entries 18 and 30). These yields are not surprising, especially since the cobalt boride mediated method has been shown to cleanly reduce this functionality to amines. ${ }^{13}$

Although this improved method for the reduction of the cyano group is reliable and tolerates some functional groups, small changes to the procedure and/or a change in metal boride can lead to a total change in reducing ability and selectivity. ${ }^{13,14}$ Further to these results in a few cases slight modification of the reaction procedure was found to improve yields dramatically. Whereas in most cases a reaction time of $15 \mathrm{~h}$ was required (e.g. 2 n $28 \%$ ( $1 \mathrm{~h}$ ), $59 \%$ $(15 \mathrm{~h})$ ) other cases show the yields could be improved by reducing the reaction time to $1 \mathrm{~h}$, in some cases avoiding further reaction of sensitive functional groups (2l 93\% (1 h), $25 \%(24 \mathrm{~h}))$. It is notable that nitriles containing $\alpha$-acidic protons were reduced in high yields under these reaction conditions and only catalytic reduction of benzyl cyanide $\mathbf{1 b}$ showed a reduced yield, which was easily overcome by addition of further sodium borohydride. One of the most significant improvements to the procedure was the introduction of diethylenetriamine, a stronger coordinating ligand for the nickel species than the Boc amines, to the work-up procedure. This improved yields and/or consistency thereof in many cases (2a (80\%, cf. 65\%), 2e (67\%, cf. $14 \%), 2 \mathrm{~m}(87 \%$, cf. $60 \%)){ }^{31}$ Although some starting compounds, such as pyridines, were also found to cause slow decomposition of the metal boride, this process was generally slow enough to allow complete conversion to the Boc amine. Investigations directed towards further improvement of this procedure by employing polymer 
Table 1. Catalytic reduction of nitriles to Boc amines

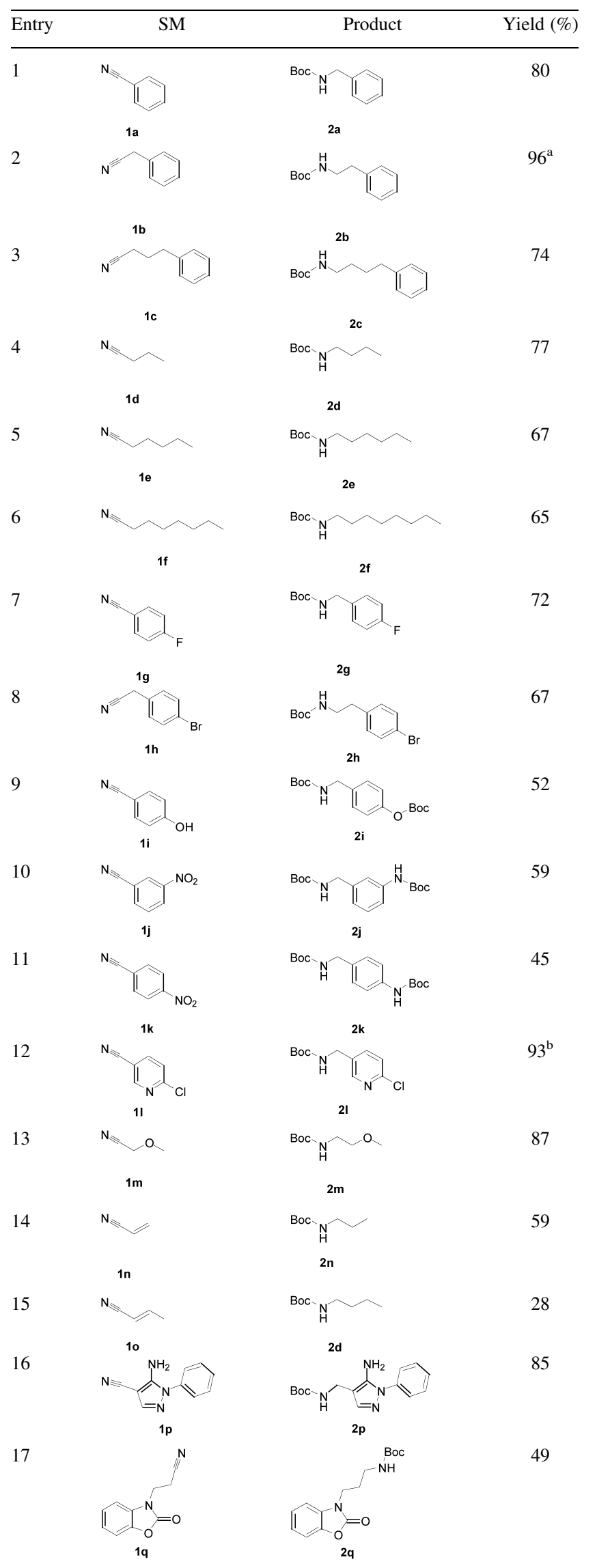

Table 1 (continued)

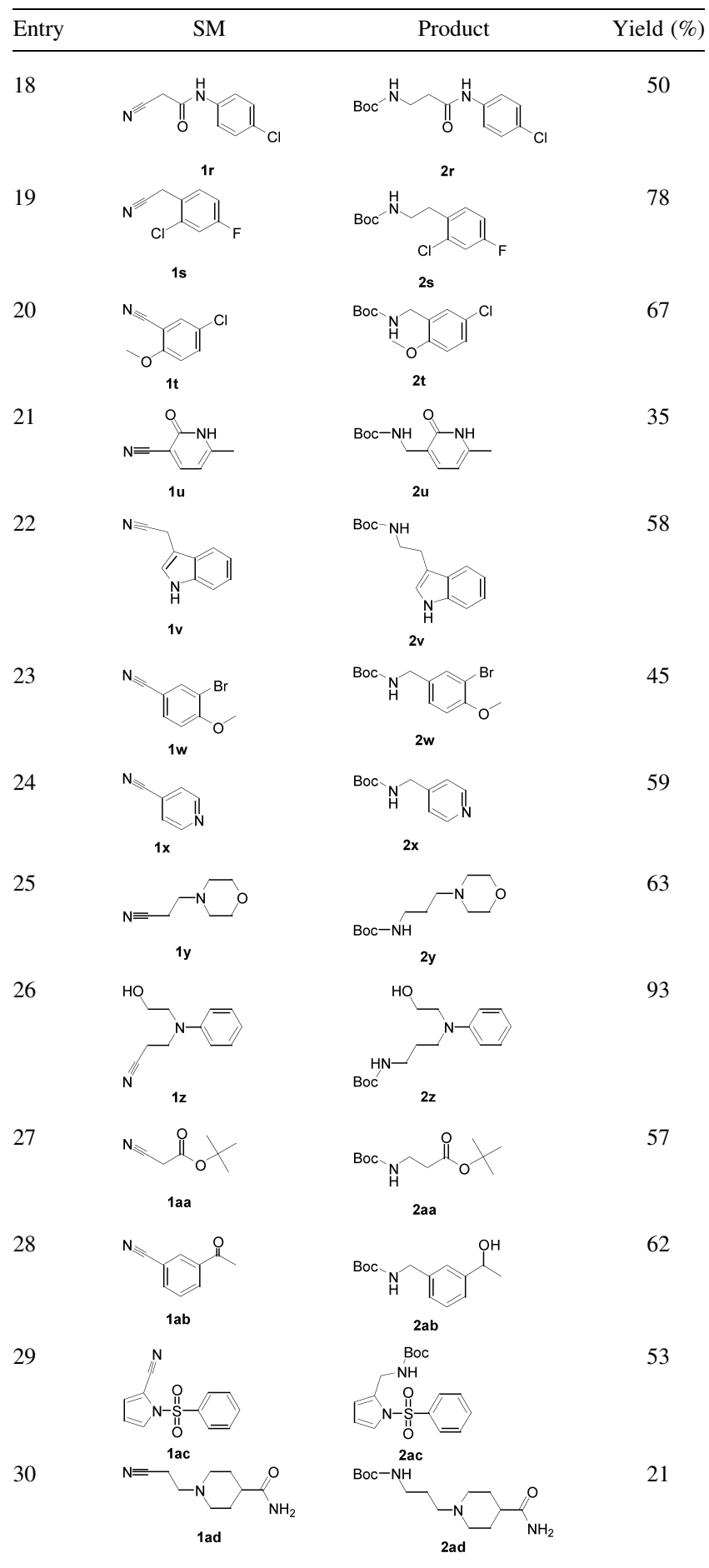

${ }^{a}$ Additional $\mathrm{NaBH}_{4}$ (4 equiv.) was added after $4 \mathrm{~h}$.

b The reaction time was reduced to $1 \mathrm{~h}$.

supported borohydride (Amberlite ${ }^{\circledR}$ IRA-400) ${ }^{32}$ suggested interference of the resin with the nickel species resulting in its decomposition.

\section{Conclusion}

In summary, a practical procedure for the catalytic reduction of nitriles to Boc amines has been developed. This clean 
transformation is marked by its non-air and moisturesensitive protocol and its ease of work-up. Nickel boride has proven a versatile, simple to prepare substance with catalytic ability promoting reduction of the cyano group.

\section{Experimental}

\subsection{General}

All reactions were performed in oven-dried glassware under a nitrogen atmosphere on a Radleys Cooled Carousel ${ }^{\mathrm{TM}}$ allowing simultaneous conversion of 12 nitriles. All reagents and solvents were purchased from commercial sources and used as supplied or purified according to standard procedures; $\mathrm{MeOH}$ was distilled from $\mathrm{Mg} / \mathrm{I}_{2}$. Analytical thin layer chromatography was performed on SIL G/UV 254 plates and visualised using standard procedures. Melting points were recorded on a Gallenkamp apparatus and are uncorrected. Infrared spectroscopy was performed on a Perkin-Elmer 1710 FT-IR spectrometer as thin films or solutions. ${ }^{1} \mathrm{H}$ And ${ }^{13} \mathrm{C} \mathrm{NMR}$ spectra were recorded on a Bruker-DPX-300 spectrometer at ambient probe temperature, using residual isotopic solvent as an internal reference. Coupling constants $(J)$ are reported in Hertz $(\mathrm{Hz}) .{ }^{13} \mathrm{C}$ NMR spectra were proton decoupled and DEPT experiments were used for assignment. Mass spectrometric analysis was recorded using a Kratos MS80RF or a Bruker Daltonics F-APEX III ESI.

4.1.1. $N$-Boc-benzylamine (2a). ${ }^{21}$ Typical procedure for the reduction of a nitrile to a Boc amine. To a stirred solution of benzonitrile 1a $(204 \mu \mathrm{L}, 2.0 \mathrm{mmol})$ in dry methanol $(15 \mathrm{~mL})$, cooled to $0^{\circ} \mathrm{C}$, were added $\mathrm{Boc}_{2} \mathrm{O}$ ( $873 \mathrm{mg}, 4.0 \mathrm{mmol})$ and $\mathrm{NiCl}_{2} \cdot 6 \mathrm{H}_{2} \mathrm{O}(48 \mathrm{mg}, 0.2 \mathrm{mmol})$. $\mathrm{NaBH}_{4}$ (530 mg, $\left.14.0 \mathrm{mmol}\right)$ was then added in small portions over $30 \mathrm{~min}$. The reaction was exothermic and effervescent. The resulting reaction mixture containing a finely divided black precipitate was allowed to warm to room temperature and left to stir for a further $1 \mathrm{~h},{ }^{33}$ at which point diethylenetriamine $(216 \mu \mathrm{L}, 2.0 \mathrm{mmol})$ was added. The mixture was allowed to stir for $30 \mathrm{~min}$ before solvent evaporation. The purple residue was dissolved in EtOAc $(50 \mathrm{~mL})$ and extracted with saturated $\mathrm{NaHCO}_{3}(2 \times 50 \mathrm{~mL})$. The organic layer was dried $\left(\mathrm{MgSO}_{4}\right)$ and the solvent removed in vacuo to yield $\mathbf{2 a}$ as a white solid $(330 \mathrm{mg}$, $80 \%$ ). $\mathrm{mp} 54-55^{\circ} \mathrm{C}$ (lit. $\mathrm{mp} 52-53^{\circ} \mathrm{C},{ }^{34} \mathrm{mp} 55.5-$ $\left.56.5^{\circ} \mathrm{C}\right) .{ }^{35}$ Spectroscopic data corresponds to that reported in the literature. ${ }^{34-36}$

Catalytic reduction of all nitriles was carried out according to the same procedure as described above, on an identical scale, however, in most cases the reaction time was $15 \mathrm{~h} .{ }^{37}$

4.1.2. Phenethyl-carbamic acid tert-butyl ester (2b). ${ }^{21}$ $424 \mathrm{mg}$, 96\%; pale yellow solid; mp 58-59 ${ }^{\circ} \mathrm{C}$ (lit. $\mathrm{mp}$ $\left.59.1^{\circ} \mathrm{C}\right) .^{21}$ Spectroscopic data corresponds to that reported in the literature. ${ }^{21}$

4.1.3. (4-Phenyl-butyl)-carbamic acid tert-butyl ester (2c). ${ }^{21} 369 \mathrm{mg}$, 74\%; colourless oil; IR (film) 3340, 2979, $1695,1523,1460,1367,1251,1170,1104,757 \mathrm{~cm}^{-1} ;{ }^{1} \mathrm{H}$ NMR (300 MHz, $\left.\mathrm{CDCl}_{3}\right) \delta 7.22(\mathrm{~d}, J=6.8 \mathrm{~Hz}, 2 \mathrm{H}), 7.17-$ $7.10(\mathrm{~m}, 3 \mathrm{H}), 5.53$ (br s, 1H), 3.10 (q, $J=7.2 \mathrm{~Hz}, 2 \mathrm{H}), 2.59$ (t, $J=7.2 \mathrm{~Hz}, 2 \mathrm{H}$ ), 1.60 (quin., $J=7.2 \mathrm{~Hz}, 2 \mathrm{H}$ ), 1.47 (quin., $J=7.2 \mathrm{~Hz}, 2 \mathrm{H}), 1.40(\mathrm{~s}, 9 \mathrm{H}) ;{ }^{13} \mathrm{C} \mathrm{NMR}\left(75 \mathrm{MHz} \mathrm{CDCl}_{3}\right) \delta$ 155.9 (C), $142.1(\mathrm{C}), 128.3(2 \times \mathrm{CH}), 128.2(2 \times \mathrm{CH}), 125.7$ $(\mathrm{CH}), 78.9(\mathrm{C}), 40.3\left(\mathrm{CH}_{2}\right), 35.4\left(\mathrm{CH}_{2}\right), 29.6\left(\mathrm{CH}_{2}\right), 28.5$ $\left(\mathrm{CH}_{2}\right), 28.3\left(3 \times \mathrm{CH}_{3}\right)$; HRMS (ES) calcd for $\mathrm{C}_{30} \mathrm{H}_{46} \mathrm{~N}_{2} \mathrm{O}_{4} \mathrm{Na}$ $[2 \mathrm{M}+\mathrm{Na}]^{+}$521.3350, found 521.3382 .

4.1.4. Butyl-carbamic acid tert-butyl ester (2d). ${ }^{21} 266 \mathrm{mg}$, $77 \%$; colourless oil. Spectroscopic data corresponds to that reported in the literature. ${ }^{38,39}$

4.1.5. Hexyl-carbamic acid tert-butyl ester (2e). ${ }^{21}$ $269 \mathrm{mg}$, 67\%; colourless oil. Spectroscopic data corresponds to that reported in the literature. ${ }^{40}$

4.1.6. Octyl-carbamic acid tert-butyl ester (2f). ${ }^{21} 298 \mathrm{mg}$, $65 \%$; colourless oil. Spectroscopic data corresponds to that reported in the literature. ${ }^{41}$

4.1.7. (4-Fluoro-benzyl)-carbamic acid tert-butyl ester (2g). ${ }^{21} 324 \mathrm{mg}, 72 \%$; white crystalline solid; $\mathrm{mp} 61-62^{\circ} \mathrm{C}$ (lit. $\left.\mathrm{mp} 68-70^{\circ} \mathrm{C}\right) .^{42}$ Spectroscopic data corresponds to that reported in the literature. ${ }^{42}$

4.1.8. [2-(4-Bromo-phenyl)-ethyl]-carbamic acid tertbutyl ester (2h). ${ }^{21} 402 \mathrm{mg}, 67 \%$; pale yellow solid; mp $58-59^{\circ} \mathrm{C}$ (lit. mp $61-62^{\circ} \mathrm{C}$ )..$^{39}$ Spectroscopic data corresponds to that reported in the literature. ${ }^{39}$

4.1.9. Carbonic acid 4-(tert-butoxycarbonylaminomethyl)-phenyl ester tert-butyl ester (2i). ${ }^{21} 336 \mathrm{mg}$, $52 \%$; white crystalline solid; $\mathrm{mp} 84-85^{\circ} \mathrm{C}$; IR $\left(\mathrm{CH}_{2} \mathrm{Cl}_{2}\right)$ 3443, 3055, 2982, 1757, 1710, 1508, 1369, 1221, 1149, $1048,894 \mathrm{~cm}^{-1}$; ${ }^{1} \mathrm{H}$ NMR $\left(300 \mathrm{MHz}, \mathrm{CDCl}_{3}\right) \delta 7.28(\mathrm{~d}$, $J=8.5 \mathrm{~Hz}, 2 \mathrm{H}), 7.11(\mathrm{~d}, J=8.5 \mathrm{~Hz}, 2 \mathrm{H}), 4.84$ (br s, $1 \mathrm{H}$ [exch]), $4.28(\mathrm{~d}, J=5.8 \mathrm{~Hz}, 2 \mathrm{H}), 1.55(\mathrm{~s}, 9 \mathrm{H}), 1.45(\mathrm{~s}, 9 \mathrm{H})$; ${ }^{13} \mathrm{C} \mathrm{NMR}\left(75 \mathrm{MHz}, \mathrm{CDCl}_{3}\right) \delta 155.8(\mathrm{C}), 151.9(\mathrm{C}), 150.2(\mathrm{C})$, $136.5(\mathrm{C}), 128.5(2 \times \mathrm{CH}), 121.4(2 \times \mathrm{CH}), 83.5(\mathrm{C}), 79.5(\mathrm{C})$, $44.1\left(\mathrm{CH}_{2}\right), 28.4\left(3 \times \mathrm{CH}_{3}\right), 27.7\left(3 \times \mathrm{CH}_{3}\right)$; HRMS (ES) calcd for $\mathrm{C}_{17} \mathrm{H}_{25} \mathrm{NO}_{5} \mathrm{Na}[\mathrm{M}+\mathrm{Na}]^{+} 346.1625$, found 346.1629 .

4.1.10. [3-(tert-Butoxycarbonylamino-methyl)-phenyl]carbamic acid tert-butyl ester (2j). ${ }^{21} 380 \mathrm{mg}$, 59\%; white solid; $\mathrm{mp} 136-137^{\circ} \mathrm{C}$; IR $\left(\mathrm{CH}_{2} \mathrm{Cl}_{2}\right) 3430,3054$, $1641,1421,1159,896 \mathrm{~cm}^{-1} ;{ }^{1} \mathrm{H}$ NMR $\left(300 \mathrm{MHz}, \mathrm{CDCl}_{3}\right) \delta$ 7.32 (br s, $1 \mathrm{H}), 7.22(\mathrm{~d}, J=5.1 \mathrm{~Hz}, 2 \mathrm{H}), 6.95(\mathrm{t}, J=5.1 \mathrm{~Hz}$, 1H), 6.55 (br s, 1H [exch]), 4.86 (br s, 1H [exch]), 4.27 (d, $J=5.8 \mathrm{~Hz}, 2 \mathrm{H}), 1.51(\mathrm{~s}, 9 \mathrm{H}), 1.45(\mathrm{~s}, 9 \mathrm{H}) ;{ }^{13} \mathrm{C}$ NMR $\left(75 \mathrm{MHz}, \mathrm{CDCl}_{3}\right) \delta 155.8(\mathrm{C}), 152.7(\mathrm{C}), 139.9(\mathrm{C}), 138.6$ (C), $129.2(2 \times \mathrm{CH}), 122.0(\mathrm{CH}), 117.4(\mathrm{CH}), 80.5(\mathrm{C}), 79.5$ (C), $44.6\left(\mathrm{CH}_{2}\right), 28.4\left(3 \times \mathrm{CH}_{3}\right), 28.3\left(3 \times \mathrm{CH}_{3}\right) ; \mathrm{MS}(\mathrm{EI}) \mathrm{m} / \mathrm{z}$ (relative intensity) $322\left(\left[\mathrm{M}^{+}\right], 14\right), 265\left(\left[\mathrm{MH}^{+}-t\right.\right.$-Bu], 6), $222\left(\left[\mathrm{M}^{+}-\mathrm{NHCO}_{2} t-\mathrm{Bu}\right], 13\right), 210(60), 193\left(\left[\mathrm{M}^{+}-\mathrm{CH}_{2-}\right.\right.$ $\left.\left.\mathrm{NHCO}_{2} t-\mathrm{Bu}\right], 35\right), 166(100), 150\left(\left[\mathrm{M}^{+}-2 \times \mathrm{CO}_{2} t-\mathrm{Bu}\right], 44\right)$, $121\left(\left[\mathrm{M}^{+}-2 \times \mathrm{NHCO}_{2} t\right.\right.$-Bu], 57), 106 (34), 93 (15), 78 (18), 57 (100); HRMS (ES) calcd for $\mathrm{C}_{17} \mathrm{H}_{26} \mathrm{~N}_{2} \mathrm{O}_{4} \mathrm{Na}[\mathrm{M}+\mathrm{Na}]^{+}$ 345.1785 , found 345.1793 .

4.1.11. [4-(tert-Butoxycarbonylamino-methyl)-phenyl]carbamic acid tert-butyl ester (2k). ${ }^{21} 290 \mathrm{mg}$, 45\%; yellow solid; $\mathrm{mp} 155-157^{\circ} \mathrm{C}$ (lit. $\mathrm{mp} 161^{\circ} \mathrm{C}$ ). ${ }^{40}$ Spectroscopic data corresponds to that reported in the literature. ${ }^{40}$ 
4.1.12. (6-Chloro-pyridin-3-ylmethyl)-carbamic acid tert-butyl ester (2l). ${ }^{21} 451 \mathrm{mg}, 93 \%$; white solid; mp $43-$ $44^{\circ} \mathrm{C}$; IR $\left(\mathrm{CH}_{2} \mathrm{Cl}_{2}\right) 3353,2976,2932,1701,1513,1454$, 1365, 1249, 1171, 749, $699 \mathrm{~cm}^{-1}$; ${ }^{1} \mathrm{H}$ NMR $(300 \mathrm{MHz}$, $\left.\mathrm{CDCl}_{3}\right) \delta 8.22(\mathrm{~d}, J=2.2 \mathrm{~Hz}, 1 \mathrm{H}), 7.54(\mathrm{dd}, J=8.0,2.2 \mathrm{~Hz}$, $1 \mathrm{H}), 7.20(\mathrm{~d}, J=8.0 \mathrm{~Hz}, 1 \mathrm{H}), 5.40($ br s, $1 \mathrm{H}), 4.22(\mathrm{~d}$, $J=5.3 \mathrm{~Hz}, 2 \mathrm{H}), 1.37(\mathrm{~s}, 9 \mathrm{H}) ;{ }^{13} \mathrm{C} \mathrm{NMR}\left(75 \mathrm{MHz} \mathrm{CDCl}_{3}\right) \delta$ $155.6(\mathrm{C}), 149.9(\mathrm{C}), 148.3(\mathrm{CH}), 137.8(\mathrm{CH}), 133.6(\mathrm{C})$, $123.8(\mathrm{CH}), 79.6(\mathrm{C}), 41.0\left(\mathrm{CH}_{2}\right), 27.9\left(3 \times \mathrm{CH}_{3}\right)$; $\mathrm{MS}(\mathrm{EI})$ $\mathrm{m} / \mathrm{z}$ (relative intensity) $243\left(\left[\mathrm{MH}^{+}\right], 44\right), 186\left(\left[\mathrm{MH}^{+}-t-\right.\right.$ $\mathrm{Bu}], 64), 169$ ([M+- $\left.\left.\mathrm{M}^{+} \mathrm{t}-\mathrm{Bu}\right], 26\right), 152$ (59), 107 (35), 84 (76), 57 (84), 49 (100); HRMS (ES) calcd for $\mathrm{C}_{11} \mathrm{H}_{16} \mathrm{~N}_{2} \mathrm{O}_{2} \mathrm{Cl}[\mathrm{M}+\mathrm{H}]^{+}$243.0895, found 243.0897.

4.1.13. (2-Methoxy-ethyl)-carbamic acid tert-butyl ester (2m). ${ }^{21} 305 \mathrm{mg}$, 87\%; colourless oil. Spectroscopic data corresponds to that reported in the literature. ${ }^{43}$

4.1.14. Propyl-carbamic acid tert-butyl ester (2n). ${ }^{21}$ $188 \mathrm{mg}$, 59\%; colourless oil. Spectroscopic data corresponds to that reported in the literature. ${ }^{39,44}$

4.1.15. (5-Amino-1-phenyl-1H-pyrazol-4-ylmethyl)-carbamic acid tert-butyl ester (2p). $490 \mathrm{mg}, 85 \%$; yellow solid; mp $86-87^{\circ} \mathrm{C}$; IR $\left(\mathrm{CH}_{2} \mathrm{Cl}_{2}\right) 3328,2977,1687,1626$, 1498, 1251, 1168, 1012, $859 \mathrm{~cm}^{-1}$; ${ }^{1} \mathrm{H}$ NMR $(300 \mathrm{MHz}$, $\left.\mathrm{CDCl}_{3}\right) \delta 7.54(\mathrm{dd}, J=7.8,1.5 \mathrm{~Hz}, 2 \mathrm{H}), 7.45(\mathrm{td}, J=7.8$, $1.7 \mathrm{~Hz}, 2 \mathrm{H}), 7.33(\mathrm{tt}, J=7.8,1.5 \mathrm{~Hz}, 1 \mathrm{H}), 7.29(\mathrm{~s}, 1 \mathrm{H}), 5.09$ (br s, 1H), 4.61 (br s, 2H), $4.05(\mathrm{~d}, J=6.2 \mathrm{~Hz}, 2 \mathrm{H}), 1.42$ (s, 9H); ${ }^{13} \mathrm{C}$ NMR $\left(75 \mathrm{MHz}, \mathrm{CDCl}_{3}\right) \delta 157.2(\mathrm{C}), 144.1(\mathrm{C})$, $139.9(\mathrm{CH}), 138.7(\mathrm{C}), 129.3(2 \times \mathrm{CH}), 127.2(\mathrm{CH}), 123.6$ $(2 \times \mathrm{CH}), 102.0(\mathrm{C}), 79.6(\mathrm{C}), 33.7\left(\mathrm{CH}_{2}\right), 27.8\left(3 \times \mathrm{CH}_{3}\right) ; \mathrm{MS}$ (EI) $\mathrm{m} / \mathrm{z}$ (relative intensity) $288\left(\left[\mathrm{M}^{+}\right], 14\right), 232\left(\left[\mathrm{MH}^{+}-t-\right.\right.$ $\mathrm{Bu}], 36), 187\left(\left[\mathrm{M}^{+}-\mathrm{CO}_{2} t-\mathrm{Bu}\right], 21\right), 173\left(\left[\mathrm{M}^{+}-\mathrm{NCO}_{2} t-\right.\right.$ $\mathrm{Bu}], 67), 145\left(\left[\mathrm{M}^{+}-\mathrm{CH}_{2} \mathrm{NHCO}_{2} t-\mathrm{Bu}-\mathrm{NH}_{2}\right], 12\right), 77$ (41), 57 (100); HRMS (ES) calcd for $\mathrm{C}_{15} \mathrm{H}_{21} \mathrm{~N}_{4} \mathrm{O}_{2}[\mathrm{M}+\mathrm{H}]^{+}$ 289.1664 , found 289.1663 .

4.1.16. [3-(5-Ethylidene-4-methylene-2-oxo-oxazolidin3-yl)-propyl]-carbamic acid tert-butyl ester (2q). $286 \mathrm{mg}$, 49\%; white crystalline solid; $\mathrm{mp} 101-103^{\circ} \mathrm{C}$; IR $\left(\mathrm{CH}_{2} \mathrm{Cl}_{2}\right)$ 3366, 2930, 1776, 1705, 1488, 1366, 1169, 1012, $925 \mathrm{~cm}^{-1} ;{ }^{1} \mathrm{H}$ NMR $\left(300 \mathrm{MHz}, \mathrm{CDCl}_{3}\right) \delta 7.21$ (dt, $J=7.6$, $1.4 \mathrm{~Hz}, 1 \mathrm{H}), 7.18(\mathrm{td}, J=7.6,1.4 \mathrm{~Hz}, 1 \mathrm{H}), 7.11(\mathrm{td}, J=7.6$, $1.4 \mathrm{~Hz}, 1 \mathrm{H}), 6.98$ (app dd, $J=7.6,1.4 \mathrm{~Hz}, 1 \mathrm{H}), 5.08$ (br s, $1 \mathrm{H}$ [exch]), $3.90(\mathrm{t}, J=6.6 \mathrm{~Hz}, 2 \mathrm{H}), 3.17(\mathrm{q}, J=6.6 \mathrm{~Hz}, 2 \mathrm{H})$, 1.95 (quin., $J=6.6 \mathrm{~Hz}, 2 \mathrm{H}), 1.42(\mathrm{~s}, 9 \mathrm{H}) ;{ }^{13} \mathrm{C} \mathrm{NMR}$ $\left(75 \mathrm{MHz}, \mathrm{CDCl}_{3}\right) \delta 156.0(\mathrm{C}), 154.9(\mathrm{C}), 142.7(\mathrm{C}), 130.8$ (C), $123.9(\mathrm{CH}), 122.6(\mathrm{CH}), 110.1(\mathrm{CH}), 108.2(\mathrm{CH}), 79.3$ (C), $39.4\left(\mathrm{CH}_{2}\right), 37.2\left(\mathrm{CH}_{2}\right), 28.3\left(3 \times \mathrm{CH}_{3}\right), 27.8\left(\mathrm{CH}_{3}\right) ; \mathrm{MS}$ (EI) $\mathrm{m} / \mathrm{z}$ (relative intensity) $292\left(\left[\mathrm{M}^{+}\right], 25\right), 236\left(\left[\mathrm{MH}^{+}-t-\right.\right.$ $\mathrm{Bu}], 100), 219\left(\left[\mathrm{M}^{+}-\mathrm{O} t-\mathrm{Bu}\right], 32\right), 191\left(\left[\mathrm{M}^{+}-\mathrm{CO}_{2} t-\mathrm{Bu}\right]\right.$, $23), 175\left(\left[\mathrm{M}^{+}-\mathrm{H}_{2} \mathrm{NCO}_{2} t-\mathrm{Bu}\right], 21\right), 162\left(\left[\mathrm{M}^{+}-\mathrm{CH}_{2-}\right.\right.$ $\left.\left.\mathrm{HNCO}_{2} t-\mathrm{Bu}\right], 30\right) 148\left(\left[\mathrm{M}^{+}-\left(\mathrm{CH}_{2}\right)_{2} \mathrm{HNCO} 2 t-\mathrm{Bu}\right], 25\right)$, $135\left(\left[\mathrm{MH}^{+}-\left(\mathrm{CH}_{2}\right)_{3} \mathrm{HNCO}_{2} t-\mathrm{Bu}\right], 24\right), 121$ (16), 102 (19), 77 (18), 57 (100); HRMS (ES) calcd for $\mathrm{C}_{15} \mathrm{H}_{21} \mathrm{~N}_{2} \mathrm{O}_{4}$ $[\mathrm{M}+\mathrm{H}]^{+}$293.1496, found 293.1499.

4.1.17. [2-(4-Chloro-phenylcarbamoyl)-ethyl]-carbamic acid tert-butyl ester (2r). ${ }^{45} 299 \mathrm{mg}, 50 \%$; pale yellow solid; $\mathrm{mp} 150-151^{\circ} \mathrm{C}$; IR $\left(\mathrm{CH}_{2} \mathrm{Cl}_{2}\right) 3465,1698,1528,1493$, 1249, 1173, 1031, $831 \mathrm{~cm}^{-1}$; ${ }^{1} \mathrm{H}$ NMR (300 MHz, MeOH$\left.d_{4}\right) \delta 7.60(\mathrm{dd}, J=9.0,2.5 \mathrm{~Hz}, 2 \mathrm{H}), 7.36(\mathrm{dt}, J=9.0,2.5 \mathrm{~Hz}$,
$1 \mathrm{H}), 7.32(\mathrm{dt}, J=9.0,2.5 \mathrm{~Hz}, 1 \mathrm{H}), 3.42(\mathrm{t}, J=6.8 \mathrm{~Hz}, 2 \mathrm{H})$, $2.58(\mathrm{t}, J=6.8 \mathrm{~Hz}, 2 \mathrm{H}), 1.46(\mathrm{~s}, 9 \mathrm{H}) ;{ }^{13} \mathrm{C} \mathrm{NMR}(75 \mathrm{MHz}$, $\left.\mathrm{MeOH}-d_{4}\right) \delta 172.7(\mathrm{C}), 158.8(\mathrm{C}), 139.1(\mathrm{C}), 130.6(\mathrm{C})$, $130.3(\mathrm{CH}), 130.1(\mathrm{CH}), 122.9(\mathrm{CH}), 122.8(\mathrm{CH}), 80.6(\mathrm{C})$, $38.6\left(\mathrm{CH}_{2}\right), 38.2\left(\mathrm{CH}_{2}\right), 29.1\left(3 \times \mathrm{CH}_{3}\right) ;$ HRMS (ES) calcd for $\mathrm{C}_{14} \mathrm{H}_{19} \mathrm{~N}_{2} \mathrm{O}_{3} \mathrm{ClNa}[\mathrm{M}+\mathrm{Na}]^{+}$321.0976, found 321.0980 .

4.1.18. [2-(2-Chloro-4-fluoro-phenyl)-ethyl]-carbamic acid tert-butyl ester (2s). $427 \mathrm{mg}, 78 \%$; white crystalline solid; mp 52-53 ${ }^{\circ} \mathrm{C}$; IR $\left(\mathrm{CH}_{2} \mathrm{Cl}_{2}\right) 3357,2978,1692,1513$, 1492, 1366, 1250, 1170, 1042, 902, $857 \mathrm{~cm}^{-1}$; ${ }^{1} \mathrm{H}$ NMR $\left(300 \mathrm{MHz}, \mathrm{CDCl}_{3}\right) \delta 7.19\left(\mathrm{dd}, J_{\mathrm{HF}}=8.2 \mathrm{~Hz}, J_{\mathrm{HH}}=6.6 \mathrm{~Hz}\right.$, $1 \mathrm{H}), 7.11\left(\mathrm{dd}, J_{\mathrm{HF}}=8.5 \mathrm{~Hz}, J_{\mathrm{HH}}=2.6 \mathrm{~Hz}, 1 \mathrm{H}\right), 6.92(\mathrm{app} \mathrm{td}$, $\left.J_{\mathrm{HF}}=8.2 \mathrm{~Hz}, J_{\mathrm{HH}}=2.6 \mathrm{~Hz}, 1 \mathrm{H}\right), 4.58($ br s, $1 \mathrm{H}), 3.35(\mathrm{q}$, $J=6.8 \mathrm{~Hz}, 2 \mathrm{H}), 2.90(\mathrm{t}, J=6.8 \mathrm{~Hz}, 2 \mathrm{H}), 1.42(\mathrm{~s}, 9 \mathrm{H}) ;{ }^{13} \mathrm{C}$ $\mathrm{NMR}\left(75 \mathrm{MHz}, \mathrm{CDCl}_{3}\right) \quad \delta \quad 162.9$ and $159.6 \quad(\mathrm{C}$, $\left.J_{\mathrm{CF}}=329 \mathrm{~Hz}\right), 155.8(\mathrm{C}), 134.7$ and $134.5\left(\mathrm{C}, J_{\mathrm{CF}}=14 \mathrm{~Hz}\right)$, $132.5(\mathrm{C}), 131.8$ and $131.7\left(\mathrm{CH}, J_{\mathrm{CF}}=11 \mathrm{~Hz}\right), 117.0$ and $116.6\left(\mathrm{CH}, J_{\mathrm{CF}}=32 \mathrm{~Hz}\right), 114.2$ and $113.9\left(\mathrm{CH}, J_{\mathrm{CF}}=28 \mathrm{~Hz}\right)$, $79.3(\mathrm{C}), 40.1\left(\mathrm{CH}_{2}\right), 33.2\left(\mathrm{CH}_{2}\right), 28.3\left(3 \times \mathrm{CH}_{3}\right)$; HRMS (ES) calcd for $\mathrm{C}_{13} \mathrm{H}_{17} \mathrm{NO}_{2} \mathrm{FClNa}[\mathrm{M}+\mathrm{Na}]^{+}$296.0830, found 296.0829 .

4.1.19. (5-Chloro-2-methoxy-benzyl)-carbamic acid tertbutyl ester (2t). $364 \mathrm{mg}$, 67\%; white solid; $\mathrm{mp} 80-82^{\circ} \mathrm{C}$; IR $\left(\mathrm{CH}_{2} \mathrm{Cl}_{2}\right)$ 3454, 2980, 1705, 1490, 1248, 1170, 908, $734 \mathrm{~cm}^{-1} ;{ }^{1} \mathrm{H} \quad \mathrm{NMR}\left(300 \mathrm{MHz}, \mathrm{CDCl}_{3}\right) \delta 7.22 \quad(\mathrm{~d}$, $J=2.5 \mathrm{~Hz}, 1 \mathrm{H}), 7.18(\mathrm{dd}, J=8.7,2.5 \mathrm{~Hz}, 1 \mathrm{H}), 6.75(\mathrm{~d}$, $J=8.7 \mathrm{~Hz}, 1 \mathrm{H}), 5.02$ (br s, $1 \mathrm{H}[\mathrm{exch}]), 4.25(\mathrm{~d}, J=6.0 \mathrm{~Hz}$, $2 \mathrm{H}), 3.81(\mathrm{~s}, 3 \mathrm{H}), 1.44(\mathrm{~s}, 9 \mathrm{H}) ;{ }^{13} \mathrm{C} \mathrm{NMR}\left(75 \mathrm{MHz}, \mathrm{CDCl}_{3}\right)$ $\delta 155.9(\mathrm{C}), 155.8(\mathrm{C}), 128.8(\mathrm{CH}), 128.7(\mathrm{C}), 128.0(\mathrm{CH})$, $125.4(\mathrm{C}), 111.3(\mathrm{CH}), 79.4(\mathrm{C}), 55.5\left(\mathrm{CH}_{3}\right), 39.9\left(\mathrm{CH}_{2}\right)$, $28.4\left(3 \times \mathrm{CH}_{3}\right) ; \mathrm{MS}(\mathrm{EI}) \mathrm{m} / \mathrm{z}$ (relative intensity) $271\left(\left[\mathrm{M}^{+}\right]\right.$, $31), 214\left(\left[\mathrm{MH}^{+}-t-\mathrm{Bu}\right], 65\right), 170\left(\left[\mathrm{M}^{+}-\mathrm{CO}_{2} t-\mathrm{Bu}\right], 81\right), 155$ $\left(\left[\mathrm{M}^{+}-\mathrm{NHCO}_{2} t-\mathrm{Bu}\right], 65\right), 136$ (53), 111 (7) 98 (10), 77 (19), 57 (100); HRMS (ES) calcd for $\mathrm{C}_{13} \mathrm{H}_{18} \mathrm{NO}_{3} \mathrm{ClNa}[\mathrm{M}+\mathrm{Na}]^{+}$ 294.0867, found 294.0868.

4.1.20. (6-Methyl-2-oxo-1,2-dihydro-pyridin-3ylmethyl)-carbamic acid tert-butyl ester (2u). $167 \mathrm{mg}$, $35 \%$; yellow crystalline solid, mp $171-172^{\circ} \mathrm{C}$; IR $\left(\mathrm{CH}_{2} \mathrm{Cl}_{2}\right)$ 3364, 2978, 1689, 1648, 1502, 1366, 1168, 1050, 907, $731 \mathrm{~cm}^{-1}$; ${ }^{1} \mathrm{H}$ NMR $\left(300 \mathrm{MHz}, \mathrm{CDCl}_{3}\right) \delta 12.82$ (br s, $1 \mathrm{H}$ [exch]), 7.35 (d, $J=6.9 \mathrm{~Hz}, 1 \mathrm{H}), 6.03(\mathrm{~d}, J=6.9 \mathrm{~Hz}, 1 \mathrm{H})$, $5.54(\mathrm{t}, J=5.7 \mathrm{~Hz}, 1 \mathrm{H}[\mathrm{exch}]), 4.14(\mathrm{~d}, J=5.7 \mathrm{~Hz}, 2 \mathrm{H}), 2.32$ $(\mathrm{s}, 3 \mathrm{H}), 1.42(\mathrm{~s}, 9 \mathrm{H}) ;{ }^{13} \mathrm{C}$ NMR $\left(75 \mathrm{MHz}, \mathrm{CDCl}_{3}\right) \delta 165.1$ (C), $156.0(\mathrm{C}), 144.3(\mathrm{C}), 139.4(\mathrm{CH}), 125.5(\mathrm{C}), 105.8$ (CH), $79.1(\mathrm{C}), 40.7\left(\mathrm{CH}_{2}\right), 28.4\left(3 \times \mathrm{CH}_{3}\right), 18.8$ (q); HRMS (ES) calcd for $\mathrm{C}_{12} \mathrm{H}_{19} \mathrm{~N}_{2} \mathrm{O}_{3}[\mathrm{M}+\mathrm{H}]^{+} 239.1390$, found 239.1393.

4.1.21. [2-(1H-Indol-3-yl)-ethyl]-carbamic acid tert-butyl ester (2v). $302 \mathrm{mg}, 58 \%$; yellow oil. Spectroscopic data corresponds to that reported in the literature. ${ }^{46}$

4.1.22. (3-Bromo-4-methoxy-benzyl)-carbamic acid tertbutyl ester (2w). $284 \mathrm{mg}$, 45\%; white crystalline solid; mp $71-73^{\circ} \mathrm{C}$; IR $\left(\mathrm{CH}_{2} \mathrm{Cl}_{2}\right) 3361,2976,1694,1498,1366,1255$, $1168,1054,1021,810 \mathrm{~cm}^{-1},{ }^{1} \mathrm{H}$ NMR $\left(300 \mathrm{MHz} \mathrm{CDCl}_{3}\right) \delta$ $7.44(\mathrm{~s}, 1 \mathrm{H}), 7.16(\mathrm{~d}, J=8.4 \mathrm{~Hz}, 1 \mathrm{H}), 6.82(\mathrm{~d}, J=8.4 \mathrm{~Hz}$, 1H), 5.00 (br s, $1 \mathrm{H}[\mathrm{exch}]), 4.20(\mathrm{~d}, J=5.5 \mathrm{~Hz}, 2 \mathrm{H}), 3.85$ (s, $3 \mathrm{H}), 1.43$ (s, 9H); ${ }^{13} \mathrm{C} \mathrm{NMR}\left(75 \mathrm{MHz}, \mathrm{CDCl}_{3}\right) \delta 155.7(\mathrm{C})$, 
$155.0(\mathrm{C}), 132.6(\mathrm{C}), 132.3(\mathrm{CH}), 127.6(\mathrm{CH}), 111.8(\mathrm{CH})$, $111.5(\mathrm{C}), 80.4(\mathrm{C}), 56.2\left(\mathrm{CH}_{3}\right), 43.4\left(\mathrm{CH}_{2}\right), 28.3\left(\mathrm{CH}_{3}\right) ; \mathrm{MS}$ (EI) $\mathrm{m} / \mathrm{z}$ (relative intensity) $317\left(\left[\mathrm{M}^{+}\right], 17\right), 315\left(\left[\mathrm{M}^{+}\right], 17\right)$, $260\left(\left[\mathrm{M}^{+}-t-\mathrm{Bu}\right], \quad 84\right), 258\left(\left[\mathrm{M}^{+}-t-\mathrm{Bu}\right], \quad 78\right), 216$ $\left(\left[\mathrm{M}^{+}-\mathrm{CO}_{2} t-\mathrm{Bu}\right], 17\right), 214\left(\left[\mathrm{M}^{+}-\mathrm{CO}_{2} t-\mathrm{Bu}\right], 19\right), 201$ $\left(\left[\mathrm{M}^{+}-\mathrm{NHCO}_{2} t-\mathrm{Bu}\right], 39\right), 199\left(\left[\mathrm{M}^{+}-\mathrm{NHCO}_{2} t-\mathrm{Bu}\right], 42\right)$, 136 (46), 77 (22), 57 (100); HRMS (ES) calcd for $\mathrm{C}_{13} \mathrm{H}_{18} \mathrm{~N}_{1} \mathrm{O}_{3} \mathrm{BrNa}[\mathrm{M}+\mathrm{Na}]^{+}$338.0362, found 338.0377.

4.1.23. Pyridin-4-ylmethyl-carbamic acid tert-butyl ester (2x). $245 \mathrm{mg}$, 59\%; pale yellow oil. Spectroscopic data corresponds to that reported in the literature. ${ }^{47}$

4.1.24. (3-Morpholin-4-yl-propyl)-carbamic acid tertbutyl ester (2y). $307 \mathrm{mg}$, 63\%; colourless oil; IR (film) 3347, 2964, 1713, 1520, 1455, 1365, 1272, 1171, 1118, $1014,864 \mathrm{~cm}^{-1} ;{ }^{1} \mathrm{H}$ NMR $\left(300 \mathrm{MHz}, \mathrm{CDCl}_{3}\right) \delta 5.53$ (br s, $1 \mathrm{H}$ [exch]), 3.72 (t, $J=4.4 \mathrm{~Hz}, 4 \mathrm{H}), 3.20$ (q, $J=5.7 \mathrm{~Hz}, 2 \mathrm{H})$, $2.43(\mathrm{~m}, 6 \mathrm{H}), 1.67$ (quin., $J=6.6 \mathrm{~Hz}, 2 \mathrm{H}), 1.45(\mathrm{~s}, 9 \mathrm{H}) ;{ }^{13} \mathrm{C}$ NMR $\left(75 \mathrm{MHz}, \mathrm{CDCl}_{3}\right) \quad \delta 155.9(\mathrm{C}), 78.6(\mathrm{C}), 66.8$ $\left(2 \times \mathrm{CH}_{2}\right), 57.0\left(\mathrm{CH}_{2}\right), 53.5\left(2 \times \mathrm{CH}_{2}\right), 39.6\left(\mathrm{CH}_{2}\right), 28.3$ $\left(3 \times \mathrm{CH}_{3}\right), 25.9\left(\mathrm{CH}_{2}\right)$; HRMS (ES) calcd for $\mathrm{C}_{12} \mathrm{H}_{25} \mathrm{~N}_{2} \mathrm{O}_{3}$ $[\mathrm{M}+\mathrm{H}]^{+} 245.1860$, found 245.1862.

4.1.25. $\{3-[(2-H y d r o x y-e t h y l)-p h e n y l-a m i n o]-p r o p y l\}-$ carbamic acid tert-butyl ester (2z). $547 \mathrm{mg}$, 93\%; colourless oil; IR (film) 3353, 2975, 1693, 1598, 1505, 1366, 1251, $1169,1037,864,748 \mathrm{~cm}^{-1} ;{ }^{1} \mathrm{H}$ NMR $\left(300 \mathrm{MHz} \mathrm{CDCl}_{3}\right) \delta$ $7.20(\mathrm{t}, J=8.0 \mathrm{~Hz}, 2 \mathrm{H}), 6.72(\mathrm{~m}, 3 \mathrm{H}), 4.80$ (br s, $1 \mathrm{H}[\mathrm{exch}])$, $3.75(\mathrm{t}, J=5.8 \mathrm{~Hz}, 2 \mathrm{H}), 3.43(\mathrm{t}, J=5.8 \mathrm{~Hz}, 2 \mathrm{H}), 3.35(\mathrm{t}$, $J=7.2 \mathrm{~Hz}, 2 \mathrm{H}), 3.17(\mathrm{q}, J=7.2 \mathrm{~Hz}, 2 \mathrm{H}), 2.59$ (br s, $1 \mathrm{H}$ [exch]), 1.76 (quin., $J=7.2 \mathrm{~Hz}, 2 \mathrm{H}), 1.44(\mathrm{~s}, 9 \mathrm{H}) ;{ }^{13} \mathrm{C} \mathrm{NMR}$ $\left(75 \mathrm{MHz}, \mathrm{CDCl}_{3}\right) \delta 156.1(\mathrm{C}), 148.2(\mathrm{C}), 129.2(2 \times \mathrm{CH})$, $116.9(\mathrm{CH}), 113.1(2 \times \mathrm{CH}), 79.2(\mathrm{C}), 59.8\left(\mathrm{CH}_{2}\right), 53.7$ $\left(\mathrm{CH}_{2}\right), 49.1\left(\mathrm{CH}_{2}\right), 38.3\left(\mathrm{CH}_{2}\right), 28.3\left(3 \times \mathrm{CH}_{3}\right), 27.5\left(\mathrm{CH}_{2}\right)$; MS (EI) $\mathrm{m} / \mathrm{z}$ (relative intensity) $294\left(\left[\mathrm{M}^{+}\right], 34\right), 263$ $\left(\left[\mathrm{M}^{+}-\mathrm{CH}_{2} \mathrm{OH}\right], \quad 66\right), 221 \quad\left(\left[\mathrm{M}^{+}-\mathrm{O} t-\mathrm{Bu}\right], \quad 45\right), 207$ $\left(\left[\mathrm{M}^{+}-\mathrm{CH}_{2} \mathrm{OH}-t-\mathrm{Bu}\right], 55\right), 189$ (22), 150 (66), 120 (95), 106 (75), 77 (44), 57 (100); HRMS (ES) calcd for $\mathrm{C}_{16} \mathrm{H}_{27} \mathrm{~N}_{2} \mathrm{O}_{3}[\mathrm{M}+\mathrm{H}]^{+}$295.2016, found 295.2014.

4.1.26. 3-tert-Butoxycarbonylamino-propionic acid isopropyl ester (2aa). $279 \mathrm{mg}, 57 \%$; colourless oil; IR (film) 3367, 2978, 1720, 1514, 1366, 1250, 1157, 1073, $845 \mathrm{~cm}^{-1}$; ${ }^{1} \mathrm{H}$ NMR (300 MHz, $\left.\mathrm{CDCl}_{3}\right) \delta 5.18$ (br s, $\left.1 \mathrm{H}[\mathrm{exch}]\right), 3.34$ $(\mathrm{q}, J=6.0 \mathrm{~Hz}, 2 \mathrm{H}), 2.42(\mathrm{t}, J=6.0 \mathrm{~Hz}, 2 \mathrm{H}), 1.44(\mathrm{~s}, 9 \mathrm{H}), 1.42$ $(\mathrm{s}, 9 \mathrm{H}) ;{ }^{13} \mathrm{C} \mathrm{NMR}\left(75 \mathrm{MHz}, \mathrm{CDCl}_{3}\right) \delta 172.0(\mathrm{C}), 155.7(\mathrm{C})$, $80.6(\mathrm{C}), 78.9(\mathrm{C}), 36.1\left(\mathrm{CH}_{2}\right), 35.5\left(\mathrm{CH}_{2}\right), 28.2\left(3 \times \mathrm{CH}_{3}\right)$, $27.9\left(3 \times \mathrm{CH}_{3}\right)$; HRMS (ES) calcd for $\mathrm{C}_{12} \mathrm{H}_{23} \mathrm{NO}_{4} \mathrm{Na}$ $[\mathrm{M}+\mathrm{Na}]^{+}$268.1519, found 268.1517 .

4.1.27. [3-(1-Hydroxy-ethyl)-benzyl]-carbamic acid tertbutyl ester (2ab). $311 \mathrm{mg}$, 62\%; colourless oil; IR (film) 3348, 2975, 1694, 1515, 1366, 1275, 1167, 1078, $791 \mathrm{~cm}^{-1}$; ${ }^{1} \mathrm{H}$ NMR $\left(300 \mathrm{MHz}, \mathrm{CDCl}_{3}\right) \delta 7.26(\mathrm{~m}, 3 \mathrm{H}), 7.16(\mathrm{~d}$, $J=6.8 \mathrm{~Hz}, 1 \mathrm{H}), 5.05$ (br s, $1 \mathrm{H}[\mathrm{exch}]), 4.85$ (q, $J=6.4 \mathrm{~Hz}$, $1 \mathrm{H}), 4.26(\mathrm{~d}, J=5.5 \mathrm{~Hz}, 2 \mathrm{H}), 2.82(\mathrm{~s}, 1 \mathrm{H}[\mathrm{exch}]), 1.47$ (d, $J=6.4 \mathrm{~Hz}, 3 \mathrm{H}), 1.46(\mathrm{~s}, 9 \mathrm{H}) ;{ }^{13} \mathrm{C} \mathrm{NMR}\left(75 \mathrm{MHz} \mathrm{CDCl}_{3}\right) \delta$ 155.9 (C), $146.3(\mathrm{C}), 139.0(\mathrm{C}), 128.6(\mathrm{CH}), 126.3(\mathrm{CH})$, $124.3(2 \times \mathrm{CH}), 79.4(\mathrm{C}), 69.9(\mathrm{CH}), 44.5\left(\mathrm{CH}_{2}\right), 27.8$ $\left(3 \times \mathrm{CH}_{3}\right), 25.1\left(\mathrm{CH}_{3}\right)$; MS (EI) $\mathrm{m} / z$ (relative intensity) 249 $\left(\left[\mathrm{M}^{+}-\mathrm{H}\right], 4\right), 194\left(\left[\mathrm{MH}^{+}-t-\mathrm{Bu}\right], 92\right), 177\left(\left[\mathrm{M}^{+}-\mathrm{O} t-\mathrm{Bu}\right]\right.$, 100), 160 (39), 150 ([ $\left.\left.\mathrm{M}^{+}-\mathrm{CO}_{2} t-\mathrm{Bu}\right], 17\right), 132$ (82), 119
(30), 106 (17), 57 (19); HRMS (ES) calcd for $\mathrm{C}_{14} \mathrm{H}_{21} \mathrm{NO}_{3} \mathrm{Na}$ $[\mathrm{M}+\mathrm{Na}]^{+} 274.1413$, found 274.1421 .

4.1.28. (1-Benzenesulfonyl- $1 H$-pyrrol-2-ylmethyl)-carbamic acid tert-butyl ester (2ac). $356 \mathrm{mg}, 53 \%$; colourless oil; IR (film) 3420, 2978, 1712, 1504, 1366, 1250, 1176, 1090, 1052, 727, $590 \mathrm{~cm}^{-1} ;{ }^{1} \mathrm{H}$ NMR (300 MHz, $\left.\mathrm{CDCl}_{3}\right) \delta$ $7.75(\mathrm{dd}, J=7.5,1.6 \mathrm{~Hz}, 2 \mathrm{H}), 7.60(\mathrm{tt}, J=7.5,1.6 \mathrm{~Hz}, 1 \mathrm{H})$, $7.50(\mathrm{t}, J=7.5 \mathrm{~Hz}, 2 \mathrm{H}), 7.25(\mathrm{dd}, J=3.2,2.0 \mathrm{~Hz}, 1 \mathrm{H}), 6.25$ (app s, 1H), $6.22(\mathrm{t}, J=3.2 \mathrm{~Hz}, 1 \mathrm{H}), 5.2$ (br s, $1 \mathrm{H}$ [exch]), $4.31(\mathrm{~d}, J=6.3 \mathrm{~Hz}, 2 \mathrm{H}), 1.40(\mathrm{~s}, 9 \mathrm{H}) ;{ }^{13} \mathrm{C} \mathrm{NMR}(75 \mathrm{MHz}$, $\left.\mathrm{CDCl}_{3}\right) \delta 155.4(\mathrm{C}), 139.1(\mathrm{C}), 133.9(\mathrm{CH}), 132.3(\mathrm{C}), 129.5$ $(2 \times \mathrm{CH}), 126.4(2 \times \mathrm{CH}), 123.2(\mathrm{CH}), 115.0(\mathrm{CH}), 112.0$ (CH), 79.4 (C), $36.8\left(\mathrm{CH}_{2}\right), 28.3\left(3 \times \mathrm{CH}_{3}\right) ; \mathrm{MS}$ (EI) $\mathrm{m} / \mathrm{z}$ (relative intensity) $279\left(\left[\mathrm{M}^{+}-t-\mathrm{Bu}\right], 84\right), 235\left(\left[\mathrm{M}^{+}-\mathrm{CO}_{2} t-\right.\right.$ $\mathrm{Bu}], 41), 220$ ([ $\left.\left.\mathrm{M}^{+}-\mathrm{NHCO}_{2} t-\mathrm{Bu}\right], 68\right), 195$ (28), 139 (100), 121 (30), 94 (57), 77 (89), 57 (90); HRMS (ES) calcd for $\mathrm{C}_{16} \mathrm{H}_{20} \mathrm{~N}_{2} \mathrm{O}_{4} \mathrm{SNa}[\mathrm{M}+\mathrm{Na}]^{+} 359.1036$, found 359.1052 .

4.1.29. [3-(4-Carbamoyl-piperidin-1-yl)-propyl]-carbamic acid tert-butyl ester (2ad). $120 \mathrm{mg}, 21 \%$; white solid; $\mathrm{mp} 123-125^{\circ} \mathrm{C}$; IR $\left(\mathrm{CH}_{2} \mathrm{Cl}_{2}\right) 3381,2929,1689$, 1651, 1524, 1449, 1365, 1280, $1171 \mathrm{~cm}^{-1}$; ${ }^{1} \mathrm{H}$ NMR $\left(300 \mathrm{MHz}, \mathrm{CDCl}_{3}\right) \delta 5.84$ (br s, 1H), 5.69 (br s, 1H), 5.40 (br s, 1H), 3.15 (q, $J=6.6 \mathrm{~Hz}, 2 \mathrm{H}), 2.93(\mathrm{dt}, J=11.7,2.5 \mathrm{~Hz}$, $2 \mathrm{H}), 2.36(\mathrm{t}, J=6.6 \mathrm{~Hz}, 2 \mathrm{H}), 2.11(\mathrm{~m}, 1 \mathrm{H}), 1.89(\mathrm{dd}, J=11.7$, $2.5 \mathrm{~Hz}, 2 \mathrm{H}), 1.82(\operatorname{app~d}, J=11.7 \mathrm{~Hz}, 2 \mathrm{H}), 1.73(\mathrm{td}, J=11.7$, $3.2 \mathrm{~Hz}, 2 \mathrm{H}$ ), 1.63 (quin., $J=6.6 \mathrm{~Hz}, 2 \mathrm{H}), 1.41$ (s, 9H); ${ }^{13} \mathrm{C}$ NMR (75 MHz, $\left.\mathrm{CDCl}_{3}\right) \delta 177.5(\mathrm{C}), 156.1(\mathrm{C}), 78.8(\mathrm{C})$, $56.8\left(\mathrm{CH}_{2}\right), 53.2\left(2 \times \mathrm{CH}_{2}\right), 42.7(\mathrm{CH}), 39.8\left(\mathrm{CH}_{2}\right), 28.9$ $\left(2 \times \mathrm{CH}_{2}\right), 28.4\left(3 \times \mathrm{CH}_{3}\right), 26.5\left(\mathrm{CH}_{2}\right) ; \mathrm{MS}(\mathrm{EI}) \mathrm{m} / \mathrm{z}$ (relative intensity) $285\left(\left[\mathrm{M}^{+}\right], 21\right), 228\left(\left[\mathrm{M}^{+}-t-\mathrm{Bu}\right], 29\right), 212$ $\left(\left[\mathrm{M}^{+}-\mathrm{O} t-\mathrm{Bu}\right], \quad 74\right), 167\left(\left[\mathrm{M}^{+}-\mathrm{NHCO}_{2} t-\mathrm{Bu}\right], 38\right), 155$ $\left(\left[\mathrm{M}^{+}-\mathrm{CH}_{2} \mathrm{NHCO}_{2} t-\mathrm{Bu}\right], 63\right), 142\left(\left[\mathrm{M}^{+}-\left(\mathrm{CH}_{2}\right)_{2} \mathrm{NHCO}_{2} t-\right.\right.$ $\mathrm{Bu}], 100), 127\left(\left[\mathrm{M}^{+}-\left(\mathrm{CH}_{2}\right)_{3} \mathrm{NHCO}_{2} t-\mathrm{Bu}\right], 100\right), 98$ (60), 82 (63), 57 (88); HRMS (ES) calcd for $\mathrm{C}_{14} \mathrm{H}_{28} \mathrm{~N}_{3} \mathrm{O}_{3}$ $[\mathrm{M}+\mathrm{H}]^{+}$286.2125, found 286.2129.

\section{Acknowledgements}

We gratefully acknowledge support of this project from GSK and to Radleys for the kind gift of a Cooled Carousel $^{\mathrm{TM}}$. We also acknowledge financial support from AstraZeneca, Novartis, Pfizer, EPSRC, BBSRC, and AICR. We are grateful to EPSRC Mass Spectrometry Service at Swansea and Drs Abdul-Sada, Avent and Hitchcock for their contributions to this work.

\section{References}

1. For a selection of reviews see (a) Trost, B. M.; Fleming, I. Comprehensive Organic Synthesis; Pergamon: Oxford, 1991; Vol. 8. pp 251-254. (b) Walker, E. R. H. Chem. Soc. Rev. 1976, 5, 23. (c) See for example: Klenke, B.; Gilbert, I. H. J. Org. Chem. 2001, 66, 2480.

2. Kudo, T.; Nose, A. Chem. Pharm. Bull. 1986, 34, 3905.

3. (a) Natsume, M.; Wada, M. Chem. Pharm. Bull. 1975, 23, 2567. (b) Kikugawa, Y.; Kuramoto, M.; Saito, I.; Yamada, S.I. Chem. Pharm. Bull. 1973, 21, 1927.

4. Ganem, B.; Osby, J. O. Chem. Rev. 1986, 86, 763. 
5. (a) Periasamy, M.; Thirumalaikumur, M. J. Organomet. Chem. 2000, 609, 137. (b) Carlier, P. R.; Lo, K. M.; Williams, I. D. J. Org. Chem. 1995, 60, 7511. (c) Ito, K.; Itsuno, S.; Sakurai, Y. Synthesis 1988, 995. (d) Wade, R. C. J. Mol. Catal. 1983, 18, 273. (e) Niino, Y.; Nishiki, M.; Mitsuo, N.; Miyataka, H.; Satoh, T. Tetrahedron Lett. 1982, 23, 193. (f) Hibino, S.; Kano, S.; Sugino, E.; Tanaka, Y. Synthesis 1980, 695. (g) Chung, S.-K. J. Org. Chem. 1979, 44, 1014. (h) Satoh, T.; Suzuki, S. Chem. Pharm. Bull. 1971, 19, 817. (i) Nystrom, R. F. J. Am. Chem. Soc. 1955, 77, 2544.

6. (a) Covey, D. F.; Ferrendelli, J. A.; Hill, M. W.; Hsiang, B. C. H.; Latifi, T. N.; Reddy, P. A.; Rothman, S. M.; Woodward, K. E. J. Med. Chem. 1996, 36, 1898. (b) Buchanan, J. G.; Lumbard, K. W.; Sturgeon, R. J.; Thompson, D. K.; Wightman, R. H. J. Chem. Soc., Perkin Trans. 1 1990, 699. (c) Beaulieu, P. L.; Schiller, P. W. Tetrahedron Lett. 1988, 29, 2019. (d) Ganem, B.; Heinzman, S. W.; Osby, J. O. J. Am. Chem. Soc. 1986, 108, 67. (e) Ganem, B.; Heinzman, S. W. J. Am. Chem. Soc. 1982, 104, 6801.

7. Kunesch, N.; Lu, Y.; Miet, C.; Poisson, J. Tetrahedron: Asymmetry 1990, 1, 707.

8. (a) Schlesinger, H. I.; Brown, H. C.; Finholt, E.; Gilbreath, J. R.; Hoekstra, H. R.; Hyde, E. K. J. Am. Chem. Soc. 1953, 75, 2156. (b) Schlesinger, H. I.; Brown, H. C. USP 1949, 2461661.

9. Encyclopaedia of Reagents for Organic Synthesis; Paquette, L. A., Ed.; Wiley: Chichester, 1995; Vol. 2, pp 3694-3699.

10. Yakabe,; Belisle, C. M.; Young, Y. M.; Singaram, B. Tetrahedron Lett. 1994, 35, 5595.

11. (a) Brown, C. A. J. Org. Chem. 1970, 35, 1900. (b) Brown, L. A.; Brown, H. C. J. Am. Chem. Soc. 1963, 85, 1003.

12. Nose, A.; Kudo, T. Chem. Pharm. Bull. 1984, 32, 2421.

13. Satoh, S.; Suzuki, S. Tetrahedron Lett. 1969, 10, 4555.

14. Nose, A.; Kudo, T. Chem. Pharm. Bull. 1989, 37, 816.

15. Nose, A.; Kudo, T. Chem. Pharm. Bull. 1981, 29, 1159.

16. (a) Okamoto, Y.; Nitta, Y.; Imanaka, T.; Teranishi, S. J. Chem. Soc., Faraday Trans. 1997, 75, 2027. (b) Schreifels, J. S.; Maybury, P. C.; Swartz, Jr. W. E. J. Org. Chem. 1981, 46, 1263.

17. Hay, R. C.; Cornelius, J. E.; Russell, T. W. J. Org. Chem. 1972, 37, 3552 .

18. (a) Wang, F.; Chiba, K.; Tada, M. J. Chem. Soc., Perkin Trans. 1 1992, 1897. (b) Sato, T.; Nanba, K.; Suzuki, S. Chem. Pharm. Bull. 1971, 19, 817.

19. Osby, J. O.; Ganem, B. Tetrahedron Lett. 1985, 26, 6413.

20. Nose, A.; Kudo, T. Chem. Pharm. Bull. 1988, 36, 1529.

21. Caddick, S.; Haynes, A. K. de K.; Judd, D. B.; Williams, M. R. V. Tetrahedron Lett. 2000, 41, 3513.

22. (a) Sim, T. B.; Yoon, N. M. Bull. Chem. Soc. Jpn 1997, 70, 1101. (b) Dijkink, J.; Eriksen, K.; Goubitz, K.; Hiemstra, H.; van Zanden, M. N. A. Tetrahedron: Asymmetry 1996, 7, 515.

23. (a) Boon, D.; Darling, L. E.; Jacoby, R. L. J. Med. Chem. 1981, 24, 218. (b) Stevens, T. E. J. Org. Chem. 1960, 25, 1658.
24. Khurana, J. M.; Kukreja, G. Synth. Commun. 2002, 32, 1265.

25. Commercially available, fresh P-1 and old P-2 nickel boride were found to be ineffective.

26. Cavelier, F.; Enjalbal, C. Tetrahedron Lett. 1996, 37, 5131.

27. Seltzman, H. H.; Berrang, B. D. Tetrahedron Lett. 1993, 34, 3083.

28. Umino, N.; Iwakuma, T.; Itoh, N. Tetrahedron Lett. 1976, 13, 2875.

29. Yakabe, S.; Hirano, M.; Morimoto, T. Tetrahedron Lett. 2000, 41, 6795.

30. Belisle, C. M.; Young, Y. M.; Singaram, B. Tetrahedron Lett. 1994, 35, 5595.

31. Standard work-up procedure without addition of diethylenetriamine.

32. (a) Joung, M. J.; Ahn, J. H.; Lee, D. W.; Yoon, N. M. J. Org. Chem. 1998, 63, 2755. (b) Sim, T. B.; Choi, J.; Joung, M. J.; Yoon, N. M. J. Org. Chem. 1997, 62, 2357.

33. Reaction time for $\mathbf{2 a}, \mathbf{2 e}$ and $\mathbf{2 l} 1 \mathrm{~h}$, for all other nitriles $15 \mathrm{~h}$.

34. Wu, S.; Lee, S.; Beak, P. J. Am. Chem. Soc. 1996, 118, 715.

35. Kanazawa, A. M.; Correa, A.; Denis, J.-N.; Luche, M.-J.; Greene, A. E. J. Org. Chem. 1993, 58, 255.

36. (a) Chandrasekhar, S.; Reddy, M. V.; Chandraiah, L. Syn. Lett. 2000, 9, 1351. (b) Alonso, E.; Ramon, D. J.; Yus, M. Tetrahedron 1997, 53, 14355.

37. $\mathbf{2 a}, \mathbf{2} \mathbf{e}$ and $\mathbf{2 l}$ were an exception. Here the reaction time was reduced to $1 \mathrm{~h}$.

38. (a) Kim, Y. H.; Park, H. S. Syn. Lett. 1998, 3, 261. (b) Greck, C.; Bischoff, L.; Girard, A.; Hajicek, J.; Genet, J.-P. Bull. Soc. Chim. Fr. 1994, 4, 429. (c) Menendez, E.; Gotor, V. Synthesis 1993, 72 .

39. Tanaka, K.-I.; Yoshifuji, S.; Nitta, Y. Chem. Pharm. Bull. 1988, 36, 3125.

40. Strazzolini, P.; Melloni, T.; Guimanini, A. G. Tetrahedron 2001, 57, 9033.

41. (a) Berree, F.; Bazureau, J.-P.; Michelot, G.; Le Corre, M. Synth. Commun. 1999, 29, 2685. (b) Afonso, C. A. M. Synth. Commun. 1998, 28, 261. (c) Ohta, A.; Inagawa, Y.; Yukiko, I.; Masami, S.; Makoto, M. J. Heterocycl. Chem. 1985, 22, 1173.

42. Barberis, C.; Voyer, N.; Roby, J.; Chenard, S.; Tremblay, M.; Labrie, P. Tetrahedron 2001, 57, 2965.

43. Ninomiya, K.; Shiori, T.; Yamada, S. Tetrahedron 1974, 30, 2151.

44. (a) Benalil, A.; Roby, P.; Carboni, B.; Vaultier, M. Synthesis 1991, 9, 787. (b) Kim, S.; Lee, J. I.; Yi, K. Y. Bull. Chem. Soc. Jpn 1985, 58, 3570.

45. Rotamers are observed in the aromatic region of the ${ }^{1} \mathrm{H}$ NMR spectrum.

46. Benson, S. C.; Lee, L.; Yang, L.; Snyder, J. K. Tetrahedron 2000, 56, 1165.

47. Brana, M. F.; Castellano, J. M.; Rodriguez, M. L. L.; Galvez, L.; Amil, M. R.; Rubio, E. J. Heterocycl. Chem. 1987, 24, 369. 